MATHEMATICS OF COMPUTATION

Volume 74 , Number 250 , Pages $743-763$

S 0025-5718(04)01708-9

Article electronically published on August 20, 2004

\title{
SOBOLEV BOUNDS ON FUNCTIONS WITH SCATTERED ZEROS, WITH APPLICATIONS TO RADIAL BASIS FUNCTION SURFACE FITTING
}

\author{
FRANCIS J. NARCOWICH, JOSEPH D. WARD, AND HOLGER WENDLAND
}

\begin{abstract}
In this paper we discuss Sobolev bounds on functions that vanish at scattered points in a bounded, Lipschitz domain that satisfies a uniform interior cone condition. The Sobolev spaces involved may have fractional as well as integer order. We then apply these results to obtain estimates for continuous and discrete least squares surface fits via radial basis functions (RBFs). These estimates include situations in which the target function does not belong to the native space of the RBF.
\end{abstract}

\section{INTRODUCTION}

The problem of effectively representing an underlying function based on its values sampled at finitely many distinct scattered sites $X=\left\{x_{1}, \ldots, x_{N}\right\}$ lying in a compact region $\Omega \subset \mathbb{R}^{n}$ is important and arises in many applications-neural networks, computer aided geometric design, and gridless methods for solving partial differential equations, to name a few.

There are two main ways of dealing with this problem: interpolation of the data or least squares approximation of the data. In both cases one assumes the data is generated by a function $f$ belonging to a classical Sobolev space, $W_{p}^{k}(\Omega)$. One next needs to select an interpolating or approximating subspace of functions. One choice is to use multivariate splines or finite elements. In this approach, one needs to decompose $\Omega$ into a number of subregions and interpolate or approximate by multivariate polynomials on each subregion. One then sews together the pieces in a smooth way to construct the representing surface. This is, in $\mathbb{R}^{n}$ with $n \geq 3$, a nontrivial task.

Another approach, which will be the focus of this paper, is to use radial basis functions (RBFs). An RBF is a radial function $\Phi(x)=\phi(|x|)$ that is either positive definite or conditionally positive definite on $\mathbb{R}^{n}$. Interpolants for multivariate functions sampled at scattered sites are constructed from translates of RBFs with the possible addition of a polynomial term.

Received by the editor July 21, 2003.

2000 Mathematics Subject Classification. Primary 41A25; Secondary 41A05, 41A63.

Key words and phrases. Radial basis functions, Sobolev error estimates, scattered zeros, scattered data.

Research of the first author was supported by grant DMS-0204449 from the National Science Foundation.

Research of the second author was supported by grants DMS-9971276 and DMS-0204449 from the National Science Foundation.

(C)2004 American Mathematical Society 
It was Duchon [5] who introduced a type of RBF, the thin-plate spline, which he constructed via a variational technique similar to those used to obtain ordinary splines. The error analysis he provided for thin-plate splines involved reproducing kernel Hilbert space (RKHS) methods and applied to both interpolation and least squares approximation. Subsequently, the theory of RBF interpolation evolved with seminal contributions from Micchelli [9], who introduced a wide class of functions for which interpolation of scattered data was always possible, and Madych and Nelson [6, 7, who obtained $L_{\infty}$ error estimates for RBF interpolation. Least squares approximation by RBFs was treated by de Boor, DeVore and Ron [3, 12 in the case where the underlying domain was $\mathbb{R}^{n}$ and the approximating subspace had "centers" at the scaled lattice points. In particular, the theory of least squares approximation on a compact set $\Omega$ for scattered data has not gone beyond the initial work of Duchon.

In this paper we seek to extend the work of Duchon in several directions. The original work of Duchon dealt with the globally supported thin-plate splines. The natural spaces to deal with in that setting were the integer-order Sobolev spaces (or the Beppo-Levi spaces which are Sobolev semi-normed spaces). One of the goals of this paper is to obtain similar results for the locally supported Wendland functions 15] in their natural setting of fractional order Sobolev spaces. Another aim of this paper is to extend the least squares setting estimates to functions which lie outside the RKHS as has been recently done for the case of interpolation [10.

Recall that the original Duchon estimates applied to the continuous least squares setting only. That is, one approximated functions that were defined on all of $\Omega$. We will obtain discrete least squares estimates where it is assumed the function belongs to an appropriate Sobolev space $W_{2}^{k}(\Omega)$ but is only known on a discrete subset $X$. These results are the first of their kind.

Finally we wish to provide an "intrinsic proof" of all these results which relies on basic principles.

Central to our approach will be a theorem which gives very precise Sobolev norm estimates for functions having many zeros in a domain $\Omega$. Note that the interpolation error function is an example of a function having many zeros. This same concept will be important in establishing the least squares error estimates as well. In general, we believe this theorem has applications outside the realm of RBFs. In particular, a variant of the theorem below can be used to extend to more general domains some of the interpolation error estimates found in [1, Sec. 4.4]. More precisely, the following will be established in the $\mathbb{R}^{n}$ setting.

Theorem 1.1. Let $k$ be a positive integer, $0<s \leq 1,1 \leq p<\infty, 1 \leq q \leq \infty$ and let $\alpha$ be a multi-index satisfying $k>|\alpha|+n / p$ or, for $p=1, k \geq|\alpha|+n$. Let $X \subset \Omega$ be a discrete set with mesh norm $h=h_{X, \Omega}=\sup _{x \in \Omega} \min _{x_{j} \in X}\left|x-x_{j}\right|$ where $\Omega$ is a compact set with Lipschitz boundary which satisfies an interior cone condition. If $u \in W_{p}^{k+s}(\Omega)$ satisfies $\left.u\right|_{X}=0$, then

$$
|u|_{W_{q}^{|\alpha|}(\Omega)} \leq c h^{k+s-|\alpha|-n(1 / p-1 / q)_{+}}|u|_{W_{p}^{k+s}(\Omega)},
$$

where $c$ is a constant independent of $u$ and $h$, and $(x)_{+}=x$ if $x \geq 0$ and is 0 otherwise.

Here $|u|_{W_{p}^{m}(\Omega)}$ refers to the (fractional) Sobolev semi-norm (see definitions later). A precursor of this theorem may be found in [5] Prop. 2] by Duchon, who restricted 
$\Omega$ to balls of certain radii and considered only the cases $p=2$ and $s=0$. In another direction, Madych and Potter [8] obtained a restricted version of this theorem for the case $p=q$ and for functions which vanished on the boundary of $\Omega$.

A typical application of Theorem 1.1 can be described as follows. Suppose we have an interpolation process $P_{X}: W_{p}^{k+s}(\Omega) \rightarrow V_{X}$ that maps Sobolev functions to a finite dimensional subspace of $W_{p}^{k+s}(\Omega)$ with the additional property $\left|P_{X} f\right|_{W_{p}^{k+s}(\Omega)} \leq|f|_{W_{p}^{k+s}(\Omega)}$. Then Theorem 1.1 immediately gives error estimates of the form

$$
\left|f-P_{X} f\right|_{W_{q}^{|\alpha|}(\Omega)} \leq c h^{k+s-|\alpha|-n(1 / p-1 / q)_{+}}|f|_{W_{p}^{k+s}(\Omega)} .
$$

We illustrate the above in two different cases. Probably the most prominent situation is illustrated by classical univariate splines. For example, natural cubic spline interpolants are known to minimize $|\cdot|_{W_{2}^{2}[a, b]}$ amongst all interpolants from $W_{2}^{2}[a, b]$.

The second example deals with multivariate radial basis function interpolation. In our framework the error estimates fall into two parts. Theorem 1.1 gives estimates on the interpolation error. Moreover, it is well known that radial basis function interpolants are also best approximants in certain associated reproducing kernel Hilbert spaces. Hence, if such a space coincides with an appropriate Sobolev space, the (semi-)norm of the interpolant can be bounded by the (semi-)norm of the target function.

Our new approach offers a new paradigm for radial basis function interpolation error estimates, where estimates on functions with a large zero set replaces the power function approach.

The outline of the paper proceeds as follows. The remainder of this section will include the notation and terminology critical for the rest of the paper. In Section 2 , the central results concerning Sobolev norm estimates of functions having many zeros will be obtained. In particular it will be shown that compact domains which satisfy an interior cone condition may be decomposed into smaller regions which are star-shaped with respect to a ball. The Sobolev estimates will then be obtained for these smaller regions and then will be pieced together to obtain similar results for the larger region. In Section 3, the results of the previous section will then be applied to both the continuous and discrete least squares approximation problem to derive error estimates for the case of radial basis functions.

1.1. Notation. We will need to work with a variety of Sobolev spaces. The definitions used here follow those used by Brenner and Scott [1]. Let $\Omega \subset \mathbb{R}^{n}$ be a domain. For $k \geq 0, k \in \mathbb{Z}$, and $1 \leq p<\infty$, we define the Sobolev spaces $W_{p}^{k}(\Omega)$ to be all $u$ with distributional derivatives $D^{\alpha} u \in L_{p}(\Omega),|\alpha| \leq k$. Associated with these spaces are the (semi-)norms

$$
|u|_{W_{p}^{k}(\Omega)}=\left(\sum_{|\alpha|=k}\left\|D^{\alpha} u\right\|_{L^{p}(\Omega)}^{p}\right)^{1 / p} \text { and }\|u\|_{W_{p}^{k}(\Omega)}=\left(\sum_{|\alpha| \leq k}\left\|D^{\alpha} u\right\|_{L_{p}(\Omega)}^{p}\right)^{1 / p} .
$$

The case $p=\infty$ is defined in the obvious way:

$$
|u|_{W_{p}^{k}(\Omega)}=\sup _{|\alpha|=k}\left\|D^{\alpha} u\right\|_{L_{\infty}(\Omega)} \text { and }\|u\|_{W_{\infty}^{k}(\Omega)}=\sup _{|\alpha| \leq k}\left\|D^{\alpha} u\right\|_{L_{\infty}(\Omega)}
$$


For fractional order Sobolev spaces, we use the norms below. Let $1 \leq p<\infty$, $k \geq 0, k \in \mathbb{Z}$, and let $0<s<1$. We define the fractional order Sobolev spaces $W_{p}^{\bar{k}+s}(\Omega)$ to be all $u$ for which the norms below are finite.

$$
\begin{aligned}
|u|_{W_{p}^{k+s}(\Omega)} & :=\left(\sum_{|\alpha|=k} \int_{\Omega} \int_{\Omega} \frac{\left|D^{\alpha} u(x)-D^{\alpha} u(y)\right|^{p}}{|x-y|^{n+p s}} d x d y\right)^{1 / p}, \\
\|u\|_{W_{p}^{k+s}(\Omega)} & :=\left(\|u\|_{W_{p}^{k}(\Omega)}^{p}+|u|_{W_{p}^{k+s}(\Omega)}^{p}\right)^{1 / p} .
\end{aligned}
$$

Let $X:=\left\{x_{1}, \ldots, x_{N}\right\}$ be a finite, discrete subset of $\Omega$, which we now assume to be bounded. There are three quantities that we associate with $X$ : the separation radius, the mesh norm or fill distance, and the mesh ratio. Respectively, these are given by

$$
q_{X}:=\frac{1}{2} \min _{j \neq k}\left|x_{j}-x_{k}\right|, \quad h_{X, \Omega}:=\sup _{x \in \Omega} \operatorname{dist}(x, X), \quad \text { and } \rho_{X, \Omega}:=\frac{h_{X, \Omega}}{q_{X}} .
$$

Here, $|\cdot|$ denotes the Euclidean distance on $\mathbb{R}^{n}$. The first is half the smallest distance between points in $X$, the second measures the maximum distance a point in $\Omega$ can be from any point in $X$, and the final quantity, the mesh ratio, measures to what extent points in $X$ uniformly cover $\Omega$. Frequently, when the set $\Omega$ or $X$ is understood, we will drop subscripts and write $h_{X}$ or $h$. Other notation will be introduced along the way.

\section{BOUNDS FOR FUNCTIONS WITH SCATTERED ZEROS}

In this section we obtain Sobolev bounds on functions with scattered zeros in a bounded Lipschitz domain $\Omega$ that satisfies a uniform interior cone condition. This is done in two main steps. We first obtain results for a special class of domains that are star-shaped with respect to balls. We then use a decomposition of $\Omega$ into such domains to obtain the general results.

2.1. Domains star-shaped with respect to a ball. We will first obtain our bounds for a special class of domains. Following Brenner and Scott [1, Chapter 4], we will say that a domain $\mathcal{D}$ is star-shaped with respect to a ball $B\left(x_{c}, r\right):=\{x \in$ $\left.\mathbb{R}^{n}:\left|x-x_{c}\right|<r\right\}$ if for every $x \in \mathcal{D}$, the closed convex hull of $\{x\} \cup B$ is contained in $\mathcal{D}$.

We will deal only with bounded domains. Thus, there will be a ball $B\left(x_{c}, R\right)$ that contains $\mathcal{D}$. Of course, the diameter $d_{\mathcal{D}}$ of $\mathcal{D}$ satisfies $r<d_{\mathcal{D}}<2 R$. Also, Brenner and Scott [1, Definition 4.2.16] define the chunkiness parameter $\gamma$ to be the ratio of $d_{\mathcal{D}}$ to the radius of the largest ball relative to which $\mathcal{D}$ is star-shaped. This parameter comes up in various estimates and it is useful to note that it can be bounded above; namely, we have

$$
\gamma \leq \frac{2 R}{r}
$$

Finally, such domains satisfy a simple, interior cone condition, which we now describe.

Proposition 2.1. If $\mathcal{D}$ is bounded, star-shaped with respect to $B\left(x_{c}, r\right)$ and contained in $B\left(x_{c}, R\right)$, then every $x \in \mathcal{D}$ is the vertex of a cone $C \subset \mathcal{D}$ having radius $r$ and angle $\theta=2 \arcsin \left(\frac{r}{2 R}\right)$. 
Proof. It is easy to check that when $x \in B\left(x_{c}, r\right)$, the condition is satisfied if the central axis of the cone is directed along a diameter of the ball $x \in B\left(x_{c}, r\right)$. If $x$ is outside of that ball, then consider the convex hull of $x$ and the intersection of the sphere $S\left(x,\left|x-x_{c}\right|\right)=\left\{y \in \mathbb{R}^{n}:|y-x|=\left|x_{c}-x\right|\right\}$ with $B\left(x_{c}, r\right)$. This is a cone, and, because $\mathcal{D}$ is star-shaped with respect to $B\left(x_{c}, r\right)$, it is contained in $\mathcal{D}$. Its radius is the distance from $x$ to $x_{c}$. To find its angle $\theta$, consider a triangle formed by $x, x_{c}$, and any point on $y$ in the intersection of $S\left(x,\left|x-x_{c}\right|\right)$ and the sphere $S\left(x_{c}, r\right)$. This is any isosceles triangle, since $\left|x_{c}-x\right|=|y-x|$. The angle $\angle x_{c} x y=\theta$; the side opposite this angle has length $r$. A little trigonometry then gives us that $\left|x_{c}-x\right| \sin \left(\frac{1}{2} \theta\right)=\frac{1}{2} r$. Consequently, we have $\theta=2 \arcsin \left(\frac{r}{2\left|x_{c}-x\right|}\right)$. Moreover, since $\mathcal{D} \subset B\left(x_{c}, R\right)$, we also have $\left|x_{c}-x\right| \leq R$. Thus, $\theta \geq 2 \arcsin \left(\frac{r}{2 R}\right)$. Finally, $r \leq\left|x-x_{c}\right|$ implies that the cone with vertex $x$, axis along $x_{c}-x$, and angle $\theta=2 \arcsin \left(\frac{r}{2 R}\right)$ is contained in $\mathcal{D}$.

Throughout the remainder of this section, $\mathcal{D}, r, R, \gamma, \theta$, and $x_{c}$ are related in the way described above.

2.1.1. A Bernstein inequality. What we want to do next is to prove a Bernstein inequality for polynomials restricted to $\mathcal{D}$. Let $p \in \pi_{\ell}\left(\mathbb{R}^{n}\right)$ and assume that $\nabla p$ is not identically zero. The maximum of $|\nabla p(x)|$ over $\overline{\mathcal{D}}$ occurs at some point $x_{M} \in \overline{\mathcal{D}}$. Obviously, the maximum is positive. Let $\eta=\frac{\nabla p\left(x_{M}\right)}{\left|\nabla p\left(x_{M}\right)\right|}$. Because $x_{M} \in \overline{\mathcal{D}}$, Proposition 2.1, which holds for $\overline{\mathcal{D}}$ as well as $\mathcal{D}$, implies that $x_{M}$ is the vertex of a cone $C \subset \overline{\mathcal{D}}$ having radius $r$, axis along a direction $\xi$, and angle $\theta=2 \arcsin \left(\frac{r}{2 R}\right)$. We may adjust the sign of $p$ so that $\eta \cdot \xi \geq 0$. By looking at the intersection of the cone $C$ with a plane containing $\xi$ and $\eta$, we see that there is a unit vector $\zeta$ pointing into the cone and satisfying $\eta \cdot \zeta \geq \cos (\pi / 2-\theta)=\sin (\theta)$. It follows that

$$
\left|\nabla p\left(x_{M}\right)\right|=\frac{\partial p}{\partial \eta}\left(x_{M}\right) \leq \csc (\theta) \frac{\partial p}{\partial \zeta}\left(x_{M}\right)
$$

On the other hand, for $t \in \mathbb{R}, \tilde{p}(t):=p\left(x_{M}+t \zeta\right)$ is in $\pi_{\ell}(\mathbb{R})$. In particular, it obeys the usual Bernstein inequality on $0 \leq t \leq r$ :

$$
\left|\tilde{p}^{\prime}(t)\right| \leq\left(2 \ell^{2} / r\right) \max _{t \in[0, r]}|\tilde{p}(t)| \leq\left(2 \ell^{2} / r\right)\|p\|_{L_{\infty}(\mathcal{D})} .
$$

Since $\tilde{p}^{\prime}(0)=\frac{\partial p}{\partial \zeta}\left(x_{M}\right)$, we have for all $x \in \overline{\mathcal{D}}$,

$$
|\nabla p(x)| \leq\left|\nabla p\left(x_{M}\right)\right| \leq \csc (\theta) \frac{\partial p}{\partial \zeta}\left(x_{M}\right) \leq \frac{2 \ell^{2}}{r \sin (\theta)}\|p\|_{L_{\infty}(\mathcal{D})} .
$$

Noting that $\left|\frac{\partial p}{\partial x_{j}}\right| \leq|\nabla p(x)|$ and keeping track of polynomial degrees as we differentiate, we arrive at the following result.

Proposition 2.2. With the notation and assumptions of Proposition [2.1, if $p \in$ $\pi_{\ell}\left(\mathbb{R}^{n}\right)$ and if $\alpha$ is a multi-index for which $|\alpha| \leq \ell$, then

$$
\left\|D^{\alpha} p\right\|_{L_{\infty}(\mathcal{D})} \leq \frac{2^{|\alpha|}(\ell !)^{2}}{r^{|\alpha|} \sin ^{|\alpha|}(\theta)((\ell-|\alpha|) !)^{2}}\|p\|_{L_{\infty}(\mathcal{D})} \leq\left(\frac{2 \ell^{2}}{r \sin (\theta)}\right)^{|\alpha|}\|p\|_{L_{\infty}(\mathcal{D})} .
$$




\subsubsection{Polynomial reproduction and norming sets.}

Proposition 2.3. Let $p \in \pi_{\ell}\left(\mathbb{R}^{n}\right)$ and let $\mathcal{D}$ be a bounded domain that is starshaped with respect to a ball $B\left(x_{c}, r\right)$ and also contained in a ball $B\left(x_{c}, R\right)$. If the mesh norm $h$ for $X=\left\{x_{1}, \ldots, x_{N}\right\}$ in $\mathcal{D}$ satisfies

$$
h \leq \frac{r \sin (\theta)}{4(1+\sin (\theta)) \ell^{2}},
$$

then there exist complex numbers $a_{j}(x)$ such that for any multi-index $\alpha$ with $|\alpha| \leq \ell$

$$
D^{\alpha} p(x)=\sum_{j=1}^{N} a_{j}^{\alpha}(x) p\left(x_{j}\right)
$$

where

$$
\sum_{j}\left|a_{j}^{\alpha}(x)\right| \leq \frac{2^{|\alpha|+1}(\ell !)^{2}}{r^{|\alpha|} \sin ^{|\alpha|}(\theta)((\ell-|\alpha|) !)^{2}} \leq 2\left(\frac{2 \ell^{2}}{r \sin (\theta)}\right)^{|\alpha|} .
$$

Proof. See [17, Proposition 3.6] and [11, Lemma 6.2].

Remark 2.4. The result derived in [17] is stated with $h$ taken to be the mesh norm of $X$ relative to $\mathcal{D}$. In fact, in the proof of the result, $h$ is only required to satisfy the condition that every ball $B(x, h) \subset \mathcal{D}$ contains at least one point in $X$, rather than being the mesh norm. This will be useful later.

2.1.3. Approximation with averaged Taylor polynomials. In [1, Chapter 4], Brenner and Scott discuss approximating a function $u \in W_{p}^{k}(\mathcal{D})$ by averaged Taylor polynomials $Q^{k} u \in \pi_{k-1}\left(\mathbb{R}^{n}\right)$. In this section, we briefly summarize their discussion and extend some of their results.

The averaged Taylor polynomials are defined as follows. Let $B_{\rho}$ be a ball relative to which $\mathcal{D}$ is star-shaped and having radius $\rho \geq \frac{1}{2} \rho_{\max }$, the largest radius of a ball relative to which $\mathcal{D}$ is star-shaped. In particular, we have $d_{\mathcal{D}} / \rho \leq 2 \gamma$, where $\gamma$ is the chunkiness parameter. The averaged Taylor polynomials are then given by

$$
Q^{k} u(x):=\sum_{|\alpha|<k} \frac{1}{\alpha !} \int_{B_{\rho}} D^{\alpha} u(y)(x-y)^{\alpha} \phi(y) d y .
$$

Here $\phi(y) \geq 0$ is a $C^{\infty}$ "bump" function supported on $B_{\rho}$ and satisfying both $\int_{B_{\rho}} \phi(y) d y=1$ and $\max \phi \leq C \rho^{-n}$, where $C=C_{n}$. Finally, the remainder $R^{k} u$ is defined by

$$
R^{k} u=u-Q^{k} u \text {. }
$$

The following result provides a bound on $R^{k} u$.

Proposition 2.5. For $u \in W_{p}^{k}(\mathcal{D})$, with $1<p<\infty$ and $k>n / p$ or with $p=1$ and $k \geq n$,

$$
\left\|R^{k} u\right\|_{L_{\infty}(\mathcal{D})} \leq C_{k, n, p}(1+\gamma)^{n} d_{\mathcal{D}}^{k-n / p}|u|_{W_{p}^{k}(\mathcal{D})},
$$

where $C_{k, n, p}=C_{n, p} \frac{n^{k-1}}{(k-1) !}\left(k-\frac{n}{p}\right)^{1 / p-1}$ if $p>1$ and $C_{k, n, 1}=C_{n, 1} \frac{n^{k-1}}{(k-1) !}$ if $p=1$.

Proof. See Brenner and Scott [1, Proposition 4.3.2]. We remark that we have tracked down and made explicit the dependence on $\gamma$ and $k$ of the constant $C_{k, n, \gamma, p}$ used in [1]. In the process, we employed the identity $\sum_{|\alpha|=k} \frac{k !}{\alpha !}=n^{k}$. 
To deal with fractional Sobolev spaces, we need a version of the previous result that applies when $u$ belongs to $W_{p}^{k+s}(\mathcal{D})$, where $0<s<1$. We begin with this lemma.

Lemma 2.6. For $1<p<\infty$ and $k>n / p$ or $p=1$ and $k \geq n$, if $u \in W_{p}^{k}(\mathcal{D})$, and $P \in \pi_{k}\left(\mathbb{R}^{n}\right)$, then

$$
\left\|R^{k+1} u\right\|_{L_{\infty}(\mathcal{D})} \leq C_{k, n, p}(1+\gamma)^{n} d_{\mathcal{D}}^{k-n / p}|u-P|_{W_{p}^{k}(\mathcal{D})} .
$$

Proof. We begin by noting that if $P$ is in $\pi_{k}\left(\mathbb{R}^{n}\right)$, then $Q^{k+1} P=P$; that is, $Q^{k+1}$ reproduces polynomials of degree $k$. Thus, $R^{k+1} u=R^{k+1}(u-P)$. The obvious identity $R^{k+1} u=R^{k} u+Q^{k} u-Q^{k+1} u$ then implies that

$$
R^{k+1} u=R^{k+1}(u-P)=R^{k}(u-P)+\left(Q^{k}-Q^{k+1}\right)(u-P) .
$$

By the triangle inequality and Proposition 2.5, we obtain

$$
\begin{aligned}
\left\|R^{k+1} u\right\|_{L_{\infty}(\mathcal{D})} \leq & C_{k, n, p}(1+\gamma)^{n} d_{\mathcal{D}}^{k-n / p}|u-P|_{W_{p}^{k}(\mathcal{D})} \\
& +\left\|\left(Q^{k}-Q^{k+1}\right)(u-P)\right\|_{L_{\infty}(\mathcal{D})}
\end{aligned}
$$

The second of the two terms can be estimated as follows. First, from the definition of $Q^{k}$, the fact that $\max \phi \leq C \rho^{-n}$, and the identity $\sum_{|\alpha|=k} \frac{1}{\alpha !}=\frac{n^{k}}{k !}$, we get

$$
\begin{aligned}
\left\|\left(Q^{k}-Q^{k+1}\right)(u-P)\right\|_{L_{\infty}(\mathcal{D})} & \leq \sup _{x \in \mathcal{D}} \sum_{|\alpha|=k} \int_{B_{\rho}} \frac{\phi(y)|x-y|^{k}\left|D^{\alpha}(u-P)(y)\right|}{\alpha !} d y \\
& \leq d_{\mathcal{D}}^{k} \cdot C \rho^{-n} \frac{n^{k}}{k !} \max _{|\alpha|=k} \int_{B_{\rho}}\left|D^{\alpha}(u-P)(y)\right| d y .
\end{aligned}
$$

Applying Hölder's inequality to the integral above, we see that

$$
\begin{aligned}
\int_{B_{\rho}}\left|D^{\alpha}(u-P)(y)\right| d y & \leq \operatorname{vol}\left(B_{\rho}\right)^{1-1 / p}\left\|D^{\alpha}(u-P)\right\|_{L^{p}\left(B_{\rho}\right)} \\
& \leq \operatorname{vol}\left(B_{1}\right)^{1-1 / p} \rho^{n-n / p}\left\|D^{\alpha}(u-P)\right\|_{L^{p}(\mathcal{D})} \\
& \leq \operatorname{vol}\left(B_{1}\right)^{1-1 / p} \rho^{n-n / p}|u-P|_{W_{p}^{k}(\mathcal{D})} .
\end{aligned}
$$

Combining these inequalities and using $d_{\mathcal{D}} / \rho \leq 2 \gamma$, we arrive at the estimate

$$
\left\|\left(Q^{k}-Q^{k+1}\right)(u-P)\right\|_{L_{\infty}(\mathcal{D})} \leq C \operatorname{vol}\left(B_{1}\right)^{1-1 / p} \frac{2^{n / p} n^{k}}{k !} d_{\mathcal{D}}^{k-n / p} \gamma^{n / p}|u-P|_{W_{p}^{k}(\mathcal{D})}
$$

Obviously, $\gamma^{n / p} \leq(1+\gamma)^{n}$. Consequently, putting the inequality above together with (5) yields (4).

Proposition 2.7. Let $0<s \leq 1$. For $1<p<\infty$ and $k>n / p$ or $p=1$ and $k \geq n$, if $u \in W_{p}^{k+s}(\mathcal{D})$, then

$$
\left\|R^{k+1} u\right\|_{L_{\infty}(\mathcal{D})} \leq C_{k, n, p}(1+\gamma)^{n(1+1 / p)} d_{\mathcal{D}}^{k+s-n / p}|u|_{W_{p}^{k+s}(\mathcal{D})} .
$$

Proof. The case $s=1$ is a consequence of Proposition 2.5. so we may assume that $s<1$. Let $P=Q^{k+1} u$ and note that $P \in \pi_{k}\left(\mathbb{R}^{n}\right)$. The identity,

$$
D^{\beta} Q^{m} u=Q^{m-|\beta|} D^{\beta} u,
$$


which is found in [1, Proposition 4.1.17], holds for $|\beta| \leq m-1$. In particular, if we take $\beta=\alpha,|\alpha|=k$ and $m=k+1$, then we have

$$
D^{\alpha} Q^{k+1} u=Q^{1} D^{\alpha} u=\int_{B_{\rho}} \phi(y) D^{\alpha} u(y) d y,
$$

which is of course a constant. Since $\int_{B_{\rho}} \phi(y) d y=1$, we note that

$$
D^{\alpha} u(x)-D^{\alpha} Q^{k+1} u(x)=\int_{B_{\rho}} \phi(y)\left(D^{\alpha} u(x)-D^{\alpha} u(y)\right) d y .
$$

From this, a simple manipulation, bounds on $\phi$ and $|x-y| \leq d_{\mathcal{D}}$, and Hölder's inequality, it follows that

$$
\begin{aligned}
\left|D^{\alpha} u(x)-D^{\alpha} Q^{k+1} u(x)\right| & \leq \int_{B_{\rho}} \phi(y)|x-y|^{s+n / p} \frac{\left|D^{\alpha} u(x)-D^{\alpha} u(y)\right|}{|x-y|^{s+n / p}} d y \\
& \leq C \rho^{-n} d_{\mathcal{D}}^{s+n / p} \int_{B_{\rho}} \frac{\left|D^{\alpha} u(x)-D^{\alpha} u(y)\right|}{|x-y|^{s+n / p}} d y \\
& \leq C_{n, p} d_{\mathcal{D}}^{s+n / p} \rho^{-n / p}\left\|\frac{D^{\alpha} u(x)-D^{\alpha} u(y)}{|x-y|^{s+n / p}}\right\|_{L^{p}(\mathcal{D}, d y)} .
\end{aligned}
$$

Raise both sides to the power $p$. Integrate in $x$ over $\mathcal{D}$ and sum over all $|\alpha|=k$. The result is

$$
|u-P|_{W_{p}^{k}(\mathcal{D})}^{p} \leq C_{n, p}^{p} d_{\mathcal{D}}^{s p+n} \rho^{-n} \sum_{|\alpha|=k} \int_{\mathcal{D}} \int_{\mathcal{D}} \frac{\left|D^{\alpha} u(x)-D^{\alpha} u(y)\right|^{p}}{|x-y|^{n+s p}} d y d x .
$$

The double integral on the right is just $|u|_{W_{p}^{k+s}(\mathcal{D})}^{p}$. Again using $d_{\mathcal{D}} / \rho \leq 2 \gamma$ and taking the $p^{\text {th }}$ root of both sides, we obtain

$$
|u-P|_{W_{p}^{k}(\mathcal{D})} \leq 2^{n / p} C_{n, p} d_{\mathcal{D}}^{s} \gamma^{n / p}|u|_{W_{p}^{k+s}(\mathcal{D})} .
$$

Applying Lemma 2.6 yields the result.

Corollary 2.8. Let $0<s \leq 1$. For $u \in W_{p}^{k+s}(\mathcal{D})$,

$$
\left\|D^{\alpha} u-D^{\alpha} Q^{k+1} u\right\|_{L_{\infty}(\mathcal{D})} \leq C_{k, n, p}(1+\gamma)^{n(1+1 / p)} d_{\mathcal{D}}^{k+s-|\alpha|-n / p}|u|_{W_{p}^{k+s}(\mathcal{D})},
$$

provided that $1<p<\infty$ and $k>|\alpha|+n / p$, or $p=1$ and $k \geq|\alpha|+n$.

Proof. This follows directly from Proposition 2.7 the identity (7), and the inequality $\left|D^{\alpha} u\right|_{W_{p}^{k+s-|\alpha|}(\mathcal{D})} \leq|u|_{W_{p}^{k+s}(\mathcal{D})}$.

One can use function-space interpolation theory to prove Proposition [2.7 and Corollary 2.8. Indeed, the proofs are somewhat simpler. There is a difficulty in doing this, however. The fractional Sobolev norms then also must come from interpolation of integer Sobolev spaces. While these are known to be equivalent to the intrinsic fractional norms we employ here, determining the dependence of the equivalence constants on the parameters of $\mathcal{D}$ is problematic. 
2.1.4. Sobolev bounds for functions with scattered zeros in $\mathcal{D}$. We are now ready to establish Sobolev bounds for functions with scattered zeros in $\mathcal{D}$. Suppose that $X \subset \mathcal{D}$ is finite and has a mesh norm $h$ satisfying the conditions in Proposition 2.3. In addition, with $0<s \leq 1$, suppose that $u \in W_{p}^{k+s}(\mathcal{D})$ satisfies $\left.u\right|_{X}=0$, where $k>n / p$ or, if $p=1, k \geq n$. Let $v:=u-Q^{k+1} u$. Note that if $x_{j} \in X$, $v\left(x_{j}\right)=u\left(x_{j}\right)-\left(Q^{k+1} u\right)\left(x_{j}\right)=-\left(Q^{k+1} u\right)\left(x_{j}\right)$. By Proposition 2.3, with $\ell=k$, we thus have for each $x \in \mathcal{D}$,

$$
D^{\alpha}\left(Q^{k+1} u\right)(x)=-\sum_{j=1}^{N} a_{j}^{\alpha}(x) v\left(x_{j}\right)
$$

and hence that

$$
\begin{aligned}
\left|D^{\alpha}\left(Q^{k+1} u\right)(x)\right| & \leq\left(\sum_{j=1}^{N}\left|a_{j}^{\alpha}(x)\right|\right) \max _{x_{j} \in X}\left|v\left(x_{j}\right)\right| \\
& \leq 2\left(\frac{2 k^{2}}{r \sin (\theta)}\right)^{|\alpha|}\left\|u-Q^{k+1} u\right\|_{L_{\infty}(\mathcal{D})} \\
& \leq 2\left(\frac{2 k^{2}}{r \sin (\theta)}\right)^{|\alpha|} C_{k, n, p}(1+\gamma)^{n(1+1 / p)} d_{\mathcal{D}}^{k+s-n / p}|u|_{W_{p}^{k+s}(\mathcal{D})},
\end{aligned}
$$

where the last step follows from Proposition 2.7.

Next, let $\alpha$ be a multi-index satisfying $k>|\alpha|+n / p$, or $p=1$ and $k \geq|\alpha|+n$. From Corollary 2.8 the previous inequality, and the triangle inequality, we have

$$
\left\|D^{\alpha} u\right\|_{L_{\infty}(\mathcal{D})} \leq\left\{1+2\left(\frac{2 k^{2} d_{\mathcal{D}}}{r \sin (\theta)}\right)^{|\alpha|}\right\} C_{k, n, p}(1+\gamma)^{n\left(1+\frac{1}{p}\right)} d_{\mathcal{D}}^{k+s-|\alpha|-\frac{n}{p}}|u|_{W_{p}^{k+s}(\mathcal{D})} .
$$

Now, $1 \leq \gamma \leq \frac{d_{\mathcal{D}}}{r} \leq \frac{2 R}{r}=\csc (\theta / 2), \sin (\theta / 2) \leq \sin (\theta)$, and so we have that

$$
\left\|D^{\alpha} u\right\|_{L_{\infty}(\mathcal{D})} \leq 3 C_{k, n, p} 2^{|\alpha|+n+\frac{n}{p}} k^{2|\alpha|} \csc ^{2|\alpha|+n+\frac{n}{p}}(\theta / 2) d_{\mathcal{D}}^{k+s-|\alpha|-\frac{n}{p}}|u|_{W_{p}^{k+s}(\mathcal{D})}
$$

Collecting coefficients in this expression and simplifying, we obtain the following result.

Proposition 2.9. Let $k$ be a positive integer, $1 \leq p<\infty, 0<s \leq 1$, and let $\alpha$ be a multi-index satisfying $k>|\alpha|+n / p$, or, for $p=1, k \geq|\alpha|+n$. Also, let $X \subset \mathcal{D}$ be a discrete set with mesh norm $h$ satisfying (3). If $u \in W_{p}^{k+s}(\mathcal{D})$ satisfies $\left.u\right|_{X}=0$, then

$$
\left\|D^{\alpha} u\right\|_{L_{\infty}(\mathcal{D})} \leq C_{k, n, p,|\alpha|} \csc ^{2|\alpha|+n(1+1 / p)}(\theta / 2) d_{\mathcal{D}}^{k+s-|\alpha|-n / p}|u|_{W_{p}^{k+s}(\mathcal{D})},
$$

where $C_{k, n, p,|\alpha|}=3 C_{k, n, p} 2^{|\alpha|+n(1+1 / p)} k^{2|\alpha|}$.

Corollary 2.10. Let $1 \leq q \leq \infty$. With the notation and assumptions of Proposition [2.9, we have

$$
|u|_{W_{q}^{|\alpha|}(\mathcal{D})} \leq C_{k, n, p, q,|\alpha|} \csc ^{2|\alpha|+n(1+1 / p)}(\theta / 2) d_{\mathcal{D}}^{k+s-|\alpha|+n\left(\frac{1}{q}-\frac{1}{p}\right)}|u|_{W_{p}^{k+s}(\mathcal{D})} .
$$


Proof. Since $\operatorname{card}\left\{\beta \in \mathbb{N}_{0}^{n}:|\beta|=|\alpha|\right\}=\left(\begin{array}{c}|\alpha|+n-1 \\ n-1\end{array}\right)=\mathcal{O}\left(|\alpha|^{n-1}\right)$ and $\operatorname{vol}(\mathcal{D})<C_{n} d_{\mathcal{D}}^{n}$, we find that

$$
\begin{aligned}
|u|_{W_{q}^{|\alpha|}(\mathcal{D})} & \leq\left(\begin{array}{c}
|\alpha|+n-1 \\
n-1
\end{array}\right)^{1 / q} \operatorname{vol}(\mathcal{D})^{1 / q} \max _{|\beta|=|\alpha|}\left\|D^{\beta} u\right\|_{L_{\infty}(\mathcal{D})} \\
& \leq C_{n, q,|\alpha|} d_{\mathcal{D}}^{n / q} \max _{|\beta|=|\alpha|}\left\|D^{\beta} u\right\|_{L_{\infty}(\mathcal{D})} \\
& \leq C_{k, n, p, q,|\alpha|} \csc ^{2|\alpha|+n(1+1 / p)}(\theta / 2) d_{\mathcal{D}}^{k+s-|\alpha|+n\left(\frac{1}{q}-\frac{1}{p}\right)}|u|_{W_{p}^{k+s}(\mathcal{D})} .
\end{aligned}
$$

2.2. Lipschitz domains obeying a cone condition. We will now treat a domain $\Omega \subset \mathbb{R}^{n}$ that is bounded, has a Lipschitz boundary, and satisfies an interior cone condition, where the cone has a maximum radius $R_{0}$ and angle $\varphi$. Of course, the cone condition will be obeyed if we use any radius $0<R \leq R_{0}$.

To begin, we need to cover $\Omega$ with domains that are star-shaped with respect to a ball. We will employ a construction due to Duchon [5]. Let

$$
r=\frac{R \sin (\varphi)}{2(1+\sin (\varphi))} \quad \text { and } \quad T_{r}:=\left\{t \in \frac{2 r}{\sqrt{n}} \mathbb{Z}^{n}: B(t, r) \subset \Omega\right\},
$$

where $R \leq R_{0}$. Fix $x \in \Omega$. Duchon (see the proof of [5, Proposition 1]) shows that the cone $C_{x} \subset \Omega$ associated with $x$ contains one of the balls $B(t, r)$, where $t \in \frac{2 r}{\sqrt{n}} \mathbb{Z}^{n}$. This of course implies that the set $T_{r} \neq \emptyset$ and, since $|t-x|<R$, that $C_{x} \subset B(t, R) \cap \Omega$. Moreover, the closed convex hull of $\{x\} \cup B(t, r)$ is contained in $C_{x}$, because $C_{x}$ is itself convex.

Instead of fixing $x$, we now fix $t \in T_{r}$. Let $\mathcal{D}_{t}$ be the set of all $x \in \Omega$ such that the closed convex hull of $\{x\} \cup B(t, r)$ is contained in $\Omega \cap B(t, R)$. By construction, each $\mathcal{D}_{t}$ is star-shaped with respect to $B(t, r)$. What we have shown above is that every $x \in \Omega$ is in some $\mathcal{D}_{t}$, so $\Omega \subset \bigcup_{t \in T_{r}} \mathcal{D}_{t}$. Of course, it is also true that $\mathcal{D}_{t} \subset \Omega$, so in fact we have that $\Omega=\bigcup_{t \in T_{r}} \mathcal{D}_{t}$.

This implies several useful geometric facts. We have that the diameter of $\mathcal{D}_{t}$ satisfies $d_{\mathcal{D}_{t}}<2 R$ and that the angle of the cone $\theta$ in Proposition 2.1] is related to $\varphi$ via $\theta=2 \arcsin \left(\frac{r}{2 R}\right)=2 \arcsin \left(\frac{\sin (\varphi)}{4(1+\sin (\varphi))}\right)$. Also, we have that $\# T_{r}$, the cardinality of $T_{r}$, satisfies $\# T_{r}<\operatorname{vol}(\Omega) / \operatorname{vol}(B(t, r)) \leq C_{\Omega, n, \varphi} R^{-n}$.

There is one more thing that we need. Let $\chi_{S}$ denote the characteristic function of a set $S$. Because $\mathcal{D}_{t} \subset B(t, R), \chi_{\mathcal{D}_{t}}(x) \leq \chi_{B(t, R)}(x)$ for all $x \in \mathbb{R}^{n}$. By [5. Proposition 1(iii)], there is a constant $M_{1}$, which may be taken as $M_{1}=M_{1}(\varphi, n)$, such that $\sum_{t \in T_{r}} \chi_{B(t, R)}(x) \leq M_{1}$ for all $x \in \mathbb{R}^{n}$. Consequently, $\sum_{t \in T_{r}} \chi_{\mathcal{D}_{t}}(x) \leq$ $M_{1}$. We summarize these remarks below.

Lemma 2.11. With the notation introduced above, we have the following:

(1) Each $\mathcal{D}_{t}$ is star-shaped with respect to the ball $B(t, r)$ and satisfies $B(t, r) \subseteq$ $\mathcal{D}_{t} \subseteq \Omega \cap B(t, R), d_{\mathcal{D}_{t}}<2 R$, and $\theta=2 \arcsin \left(\frac{\sin (\varphi)}{4(1+\sin (\varphi))}\right)$.

(2) $\Omega=\bigcup_{t \in T_{r}} \mathcal{D}_{t}$ and $\# T_{r}<C_{\Omega, n, \varphi} R^{-n}$.

(3) There exists a constant $M_{1}=M_{1}(\varphi, n)$ such that $\sum_{t \in T_{r}} \chi_{\mathcal{D}_{t}}(x) \leq M_{1}$ for all $x \in \mathbb{R}^{n}$.

2.2.1. Sobolev bounds for functions with scattered zeros in $\Omega$. We are now ready to obtain Sobolev bounds for functions having zeros at a finite subset $X \subset \Omega$, where 
we let $h=h_{X, \Omega}$ be the mesh norm of $X$ in $\Omega$. We will assume that $h$ satisfies the following condition:

$$
h \leq k^{-2} Q(\varphi) R_{0} \quad \text { where } Q(\varphi):=\frac{\sin (\varphi) \sin (\theta)}{8(1+\sin (\theta))(1+\sin (\varphi))}
$$

We note that $\theta=2 \arcsin \left(\frac{\sin (\varphi)}{4(1+\sin (\varphi))}\right)$, so that $Q$ only depends on $\varphi$. If this assumption holds, then we can take $R=\frac{k^{2} h}{Q(\varphi)}$, for then $R \leq R_{0}$. Moreover, from the definition of $r$ in terms of $\varphi$ and $R$ given in (8), we see that $h=\frac{r \sin (\theta)}{4 k^{2}(1+\sin (\theta))}$. Hence, $h$ satisfies (3) for $\ell=k$.

We point out that every ball $B(x, h) \subset \Omega$ contains at least one point in $X$. In particular, if we have $B(x, h) \subset \mathcal{D}_{t}$, this is still the case. By Remark 2.4, if $h=h_{X, \Omega}$ satisfies (3), then the results proved earlier all hold with this $h$.

That said, we now have the following estimate.

Theorem 2.12. Let $k$ be a positive integer, $0<s \leq 1,1 \leq p<\infty, 1 \leq q \leq \infty$, and let $\alpha$ be a multi-index satisfying $k>|\alpha|+n / p$, or $p=1$ and $k \geq|\alpha|+n$. Also, let $X \subset \Omega$ be a discrete set with mesh norm h satisfying (91). If $u \in W_{p}^{k+s}(\Omega)$ satisfies $\left.u\right|_{X}=0$, then

$$
|u|_{W_{q}^{|\alpha|}(\Omega)} \leq C_{k, n, p, q,|\alpha|, \varphi} h^{k+s-|\alpha|-n(1 / p-1 / q)_{+}}|u|_{W_{p}^{k+s}(\Omega)},
$$

where $(x)_{+}=x$ if $x \geq 0$ and is 0 otherwise.

Proof. The case $q=\infty$ follows from Proposition 2.9 and the decomposition given in Lemma 2.11, $\Omega=\bigcup_{t \in T_{r}} \mathcal{D}_{t}$. Thus, we will assume $1 \leq q<\infty$. For such $q$, the decomposition $\Omega=\bigcup_{t \in T_{r}} \mathcal{D}_{t}$ implies that we have

$$
\begin{aligned}
|u|_{W_{q}^{|\alpha|}(\Omega)} & =\left(\sum_{|\beta|=|\alpha|} \int_{\Omega}\left|D^{\beta} u(x)\right|^{q} d x\right)^{1 / q} \\
& \leq\left(\sum_{t \in T_{r}|\beta|=|\alpha|} \int_{\mathcal{D}_{t}}\left|D^{\beta} u(x)\right|^{q} d x\right)^{1 / q}=\left(\sum_{t \in T_{r}}|u|_{W_{q}^{|\alpha|}\left(\mathcal{D}_{t}\right)}^{q}\right)^{1 / q} \\
& \leq\left(\# T_{r}\right)^{\left(\frac{1}{q}-\frac{1}{p}\right)_{+}}\left(\sum_{t \in T_{r}}|u|_{W_{q}^{|\alpha|}\left(\mathcal{D}_{t}\right)}^{p}\right)^{1 / p},
\end{aligned}
$$

where $\# T_{r}$ is the cardinality of $T_{r}$ and where the last bound follows from standard inequalities relating $p$ and $q$ norms on finite dimensional spaces. Next, by this inequality and Corollary 2.10 , where we use $d_{\mathcal{D}_{t}}<2 R=2 k^{2} h / Q(\varphi)$, we obtain

$$
|u|_{W_{q}^{|\alpha|}(\Omega)} \leq C_{k, n, p, q,|\alpha|, \varphi}^{\prime}\left(\# T_{r}\right)^{\left(\frac{1}{q}-\frac{1}{p}\right)}+h^{k+s-|\alpha|+n\left(\frac{1}{q}-\frac{1}{p}\right)}\left(\sum_{t \in T_{r}}|u|_{W_{p}^{k+s}\left(\mathcal{D}_{t}\right)}^{p}\right)^{1 / p}
$$


for each $t \in T_{r}$. Now, since $\mathcal{D}_{t} \subset \Omega$, we have by Lemma 2.11

$$
\begin{aligned}
\sum_{t \in T_{r}}|u|_{W_{p}^{k+s}\left(\mathcal{D}_{t}\right)}^{p} & =\sum_{|\beta|=k} \int_{\Omega}\left(\sum_{t \in T_{r}} \chi_{\mathcal{D}_{t}}(x)\right) \int_{\mathcal{D}_{t}} \frac{\left|D^{\beta} u(x)-D^{\beta} u(y)\right|^{p}}{|x-y|^{n+s p}} d y d x \\
& \leq M_{1} \sum_{|\beta|=k} \int_{\Omega} \int_{\Omega} \frac{\left|D^{\beta} u(x)-D^{\beta} u(x)\right|^{p}}{|x-y|^{n+s p}} d y d x \\
& \leq M_{1}|u|_{W_{p}^{k+s}(\Omega)}^{p} .
\end{aligned}
$$

Putting these two inequalities together yields

$$
|u|_{W_{q}^{|\alpha|}(\Omega)} \leq M_{1}^{1 / p} C_{k, n, p, q,|\alpha|, \varphi}^{\prime}\left(\# T_{r}\right)^{\left(\frac{1}{q}-\frac{1}{p}\right)}+h^{k+s-|\alpha|+n\left(\frac{1}{q}-\frac{1}{p}\right)}|u|_{W_{p}^{k+s}(\Omega)} .
$$

Now, by part (2) of Lemma 2.11 and $R=k^{2} h / Q(\varphi)$, we see that $\# T_{r}<C h^{-n}$. Inserting this in the inequality above gives us

$$
|u|_{W_{q}^{|\alpha|}(\Omega)} \leq C_{k, n, p, q,|\alpha|, \varphi} h^{k+s-|\alpha|+n\left(\frac{1}{q}-\frac{1}{p}\right)-n\left(\frac{1}{q}-\frac{1}{p}\right)}+|u|_{W_{p}^{k+s}(\Omega)} .
$$

Using $n\left(\frac{1}{q}-\frac{1}{p}\right)-n\left(\frac{1}{q}-\frac{1}{p}\right)_{+}=-n(1 / p-1 / q)_{+}$in the previous inequality yields (10).

2.2.2. Sobolev bounds on discrete $\ell_{q}$ norms. In practical situations, bounds on continuous norms, such as those we have investigated above, are less important than bounds on discrete norms. Our aim here is to obtain estimates similar to those in Theorem 2.12, again for $u \mid X=0$, but with continuous norms replaced by the discrete ones that we now define.

Let $Y=\left\{y_{1}, \ldots, y_{M}\right\}$ be a finite subset of $\Omega$, and denote its separation radius by $q_{Y}$, its mesh norm by $h_{Y}$, and its mesh ratio by $\rho_{Y}:=h_{Y} / q_{Y}$. Let $1 \leq q \leq \infty$. (Note that $q$ is not the same quantity as $q_{Y}$.) For a continuous function $u$ defined on $\Omega$, define the norm $\ell_{q}(Y)$ by

$$
\|u\|_{\ell_{q}(Y)}=\left\{\begin{array}{cl}
\left(\frac{1}{M} \sum_{j=1}^{M}\left|u\left(y_{j}\right)\right|^{q}\right)^{1 / q} & \text { for } 1 \leq q<\infty \\
\max _{1 \leq j \leq M}\left|u\left(y_{j}\right)\right| & \text { for } q=\infty
\end{array}\right.
$$

As before, we also define $\ell_{q}(Y)$-derivative norms when $u$ is in $C^{k}(\Omega)$ and $1 \leq q<\infty$ :

$$
|u|_{w_{q}^{k}(Y)}=\left(\sum_{|\alpha|=k}\left\|D^{\alpha} u\right\|_{\ell_{q}(Y)}^{q}\right)^{1 / q} \text { and }\|u\|_{w_{q}^{k}(Y)}=\left(\sum_{|\alpha| \leq k}\left\|D^{\alpha} u\right\|_{\ell_{q}(Y)}^{q}\right)^{1 / q} .
$$

The $q=\infty$ norms are defined in the obvious way. We now state the analog of Theorem $[2.12$ for the discrete norms.

Theorem 2.13. Let $k$ be a positive integer, $0<s \leq 1,1 \leq p<\infty, 1 \leq q \leq \infty$, and let $\alpha$ be a multi-index satisfying $k>|\alpha|+n / p$, or $p=1$ and $k \geq|\alpha|+n$. Also, let $X \subset \Omega$ be a discrete set with mesh norm $h=h_{X}$ satisfying (9). Let $Y=\left\{y_{1}, \ldots, y_{M}\right\} \subset \Omega$ be a second discrete set, with $h_{Y} \leq h$. If $u \in W_{p}^{k+s}(\Omega)$ satisfies $\left.u\right|_{X}=0$, then

$$
|u|_{w_{q}^{|\alpha|}(Y)} \leq C_{k, n, p, q,|\alpha|, \varphi, \Omega} \rho_{Y}^{n / q} h^{k+s-|\alpha|-n(1 / p-1 / q)_{+}}|u|_{W_{p}^{k+s}(\Omega)},
$$


where the discrete norm on the left above is defined in (12). In particular, if $|\alpha|=0$, then

$$
\|u\|_{\ell_{q}(Y)} \leq C_{k, n, p, q, \varphi, \Omega} \rho_{Y}^{n / q} h^{k+s-n(1 / p-1 / q)_{+}}|u|_{W_{p}^{k+s}(\Omega)} .
$$

Proof. The $q=\infty$ case is a direct consequence of Theorem 2.12 and $\rho_{Y} \geq 1$. We therefore assume that $q<\infty$. Let $\mathcal{D}_{t}$ be one of the star-shaped domains from the decomposition of $\Omega$ given in Lemma 2.11. From the $L_{\infty}$ bound in Proposition 2.9. the conditions on $\mathcal{D}_{t}$ in Lemma 2.11, and the fact that $d_{\mathcal{D}_{t}} \leq 2 R=2 k^{2} h / Q(\varphi)$, we have that

$$
\sum_{y_{j} \in \mathcal{D}_{t}}\left|D^{\alpha} u\left(y_{j}\right)\right|^{q} \leq C h^{(k+s-|\alpha|) q-n q / p} \operatorname{card}\left(\mathcal{D}_{t} \cap Y\right)|u|_{W_{p}^{k+s}\left(\mathcal{D}_{t}\right)}^{q} .
$$

To estimate $\operatorname{card}\left(\mathcal{D}_{t} \cap Y\right)$, we note that every point $y_{j}$ in $\mathcal{D}_{t} \cap Y$ is the center of the ball $B\left(y_{j}, q_{Y}\right)$. Now, by construction, $\mathcal{D}_{t} \subset B(t, R)$ and $q_{Y} \leq h_{Y} \leq h \leq R$, so every $B\left(y_{j}, q_{Y}\right) \subset B(t, 2 R)$. Hence, the number of points in $\mathcal{D}_{t} \cap Y$ satisfies the bound

$$
\operatorname{card}\left(\mathcal{D}_{t} \cap Y\right) \leq \frac{\operatorname{vol}(B(t, 2 R))}{\operatorname{vol}\left(B\left(y_{j}, q_{Y}\right)\right)}=\left(\frac{2 R}{q_{Y}}\right)^{n} .
$$

Recall from the previous section that we chose $R=\frac{k^{2} h}{Q(\varphi)}$, and so we have

$$
\sum_{y_{j} \in \mathcal{D}_{t}}\left|D^{\alpha} u\left(y_{j}\right)\right|^{q} \leq q_{Y}^{-n} h^{(k+s-|\alpha|) q+n-n q / p} C^{\prime}|u|_{W_{p}^{k+s}\left(\mathcal{D}_{t}\right)}^{q},
$$

where $C^{\prime}$ depends on $n, p, q, \varphi,|\alpha|$. Sum over $t \in T_{r}$ on both sides. Since every $y_{j} \in Y$ is in at least one $\mathcal{D}_{t}$, we have

$$
\begin{aligned}
\sum_{j=1}^{M}\left|D^{\alpha} u\left(y_{j}\right)\right|^{q} & \leq \sum_{t \in T_{r}} \sum_{y_{j} \in \mathcal{D}_{t}}\left|D^{\alpha} u\left(y_{j}\right)\right|^{q} \\
& \leq q_{Y}^{-n} h^{(k+s-|\alpha|) q+n-\frac{n q}{p}} C^{\prime} \sum_{t \in T_{r}}|u|_{W_{p}^{k+s}\left(\mathcal{D}_{t}\right)}^{q} .
\end{aligned}
$$

The sum on the left above is $M\left\|D^{\alpha} u\right\|_{\ell_{q}(Y)}^{q}$. To deal with the sum on the right, note that standard inequalities relating $p$ and $q$ norms on a finite dimensional space give

$$
\sum_{t \in T_{r}}|u|_{W_{p}^{k+s}\left(\mathcal{D}_{t}\right)}^{q} \leq\left(\# T_{r}\right)^{q\left(\frac{1}{q}-\frac{1}{p}\right)}+\left(\sum_{t \in T_{r}}|u|_{W_{p}^{k+s}\left(\mathcal{D}_{t}\right)}^{p}\right)^{q / p} .
$$

The sum $\sum_{t \in T_{r}}|u|_{W_{p}^{k+s}\left(\mathcal{D}_{t}\right)}^{p}$ was dealt with in proving Theorem 2.12, where we showed that it is bounded by $M_{1}|u|_{W_{p}^{k+s}(\Omega)}^{p}$. Also, recall that $\# T_{r}<C h^{-n}$. Using these bounds in our earlier inequality and dividing by $M$, we obtain

$$
\left\|D^{\alpha} u\right\|_{\ell_{q}(Y)}^{q} \leq M^{-1} q_{Y}^{-n} h^{(k+s-|\alpha|) q+n-n q / p-n q\left(\frac{1}{q}-\frac{1}{p}\right)}+C^{\prime} C^{q} M_{1}^{q / p}|u|_{W_{p}^{k+s}(\Omega)}^{q} .
$$

Summing over all multi-indices $\alpha$ of fixed length, simplifying the exponent of $h$, and suppressing constants, we arrive at

$$
|u|_{w_{q}^{|\alpha|}(Y)}^{q} \leq M^{-1} q_{Y}^{-n} h^{(k+s-|\alpha|) q-n q\left(\frac{1}{p}-\frac{1}{q}\right)}+C^{\prime \prime}|u|_{W_{p}^{k+s}(\Omega)}^{q} .
$$

Our last task is to estimate $M$, the number of points in $Y$, from below. Since the mesh norm of $Y$ relative to $\Omega$ is $h_{Y}$, every $x \in \Omega$ is in one of the closed balls 
$\overline{B\left(y_{j}, h_{Y}\right)}$, and so their union covers $\Omega$. It follows that the number of such balls, $M$, satisfies $M \geq \operatorname{vol}(\Omega) / \operatorname{vol}\left(B\left(y_{j}, h_{Y}\right)\right)$ or, equivalently,

$$
M^{-1} \leq \frac{\operatorname{vol}\left(B\left(y_{j}, h_{Y}\right)\right)}{\operatorname{vol}(\Omega)} \leq C_{\Omega, n} h_{Y}^{n} .
$$

Insert this in (14), simplify, and collect constants. Taking the $q^{\text {th }}$ root of both sides then completes the proof.

\section{LEAST SQUARES ERROR ESTIMATES FOR RBFs}

In this section, we will apply the estimates that we obtained in the previous section to obtain error estimates for both continuous and discrete least squares RBF surface fitting in a domain $\Omega$ in $\mathbb{R}^{n}$. We make the same assumptions on $\Omega$ as we did above; namely, $\Omega$ is bounded, has a Lipschitz boundary, and satisfies an interior cone condition, where again the cone is assumed to have a maximum radius $R_{0}$ and angle $\varphi$.

\subsection{RBFs, native spaces, and least squares.}

3.1.1. Positive definite RBFs. We will concentrate on radial basis functions $\Phi$ : $\mathbb{R}^{n} \rightarrow \mathbb{R}$ that have a positive, algebraically decaying Fourier transform. To be more precise, we assume that

$$
c_{1}\left(1+\|\omega\|_{2}^{2}\right)^{-\tau} \leq \widehat{\Phi}(\omega) \leq c_{2}\left(1+\|\omega\|_{2}^{2}\right)^{-\tau}, \quad \omega \in \mathbb{R}^{n},
$$

where $c_{1}, c_{2}>0$ are some constants and $\tau>n / 2$. In this case it is well known that the native space $\mathcal{N}_{\Phi}=\mathcal{N}_{\Phi}\left(\mathbb{R}^{n}\right)$ associated to $\Phi$ is the Sobolev space

$$
W_{2}^{\tau}\left(\mathbb{R}^{n}\right):=\left\{f \in L_{2}\left(\mathbb{R}^{n}\right): \widehat{f}(\cdot)\left(1+\|\cdot\|_{2}^{2}\right)^{\tau / 2} \in L_{2}\left(\mathbb{R}^{n}\right)\right\}
$$

and the native space norm

$$
\|f\|_{\mathcal{N}_{\Phi}}^{2}:=\int_{\mathbb{R}^{n}} \frac{|\widehat{f}(\omega)|^{2}}{\widehat{\Phi}(\omega)} d \omega
$$

is obviously equivalent to the Sobolev norm

$$
\|f\|_{W_{2}^{\tau}\left(\mathbb{R}^{n}\right)}^{2}:=\left\|\widehat{f}(\cdot)\left(1+\|\cdot\|_{2}^{2}\right)^{\tau / 2}\right\|_{L_{2}\left(\mathbb{R}^{n}\right)} .
$$

Later on, we will also deal with the case of thin-plate splines. The details of treating them differ somewhat from the more usual RBF case above. So, even though their treatment is in fact easier, they will be handled separately. Until then, we assume that the RBF $\Phi$ has a Fourier transform $\widehat{\Phi}$ satisfying (15).

As is well known, the great utility in RBFs is that for any finite subset $X=$ $\left\{x_{1}, \ldots, x_{N}\right\} \subset \mathbb{R}^{n}$ and arbitrary complex numbers $\left\{d_{1}, \ldots, d_{N}\right\}$, one can find a unique function $v$ from the span of $V_{X, \Phi}=\operatorname{span}\left\{\Phi\left(x-x_{j}\right)\right\}_{j=1}^{N}$ such that $v\left(x_{j}\right)=d_{j}$, $j=1, \ldots, N$. In addition, interpolants satisfy a minimum principle. If $f$ is in the native space $\mathcal{N}_{\Phi}$ and if we let the interpolant to $f$ on $X$ from $V_{X, \Phi}$ be $I_{X} f$, then

$$
\min _{v \in V_{X, \Phi}}\|f-v\|_{\mathcal{N}_{\Phi}}=\left\|f-I_{X} f\right\|_{\mathcal{N}_{\Phi}} .
$$

In particular, since we can take $v=0$ on the left above, we also have

$$
\left\|f-I_{X} f\right\|_{\mathcal{N}_{\Phi}} \leq\|f\|_{\mathcal{N}_{\Phi}} .
$$

These observations lead to the following lemma, which we will need in the sequel. 
Lemma 3.1. Let $\tau>n / 2, f \in W_{2}^{\tau}(\Omega), X=\left\{x_{1}, x_{2}, \ldots, x_{N}\right\} \subset \Omega$, and let $I_{X} f \in V_{X, \Phi}$ be the unique function that interpolates $f$ on $X$. If $\widehat{\Phi}$ satisfies (15), then there exists a constant $C_{\Omega, \Phi}$, depending on $\Omega$ and $\Phi$, such that

$$
\left\|f-I_{X} f\right\|_{W_{2}^{\tau}(\Omega)} \leq C_{\Omega, \Phi}\|f\|_{W_{2}^{\tau}(\Omega)} .
$$

Proof. We will require extension theorems for $W_{p}^{\tau}(\Omega)$, where $\Omega$ is a bounded Lipschitz domain. For the case in which $\tau$ is a nonnegative integer we may use the extension operator $\mathfrak{E}$ constructed by Stein [14, $\S$ VI.3] to extend any $f$ in $W_{p}^{\tau}(\Omega)$ to a function defined on $W_{p}^{\tau}\left(\mathbb{R}^{n}\right)$, for $1 \leq p \leq \infty$. Brenner and Scott [1, p. 280] give a brief discussion concerning extensions for fractional Sobolev spaces (i.e., $\tau \notin \mathbb{Z}$ ). They point out that combining results of DeVore and Sharpley [4, Theorems 6.1 and 6.7] immediately yields the existence of $\mathfrak{E}$ in the fractional case as well, provided only that $1 \leq p<\infty$. In particular, $\mathfrak{E}$ exists for $p=2$, the value of $p$ we are concerned with here.

Since $\mathfrak{E} f=f$ on $\Omega$ and since the values of $\left.f\right|_{X}$ uniquely determine the interpolant from $V_{\Phi, X}$, we have that $I_{X} \mathfrak{E} f=I_{X} f$. Consequently, we obtain this chain of inequalities:

$$
\begin{aligned}
\left\|f-I_{X} f\right\|_{W_{2}^{\tau}(\Omega)} & =\left\|\mathfrak{E} f-I_{X} \mathfrak{E} f\right\|_{W_{2}^{\tau}(\Omega)} \\
& \leq\left\|\mathfrak{E} f-I_{X} \mathfrak{E} f\right\|_{W_{2}^{\tau}\left(\mathbb{R}^{n}\right)} \\
& \leq c_{2}^{-1 / 2}\left\|\mathfrak{E} f-I_{X} \mathfrak{E} f\right\|_{\mathcal{N}_{\Phi}} \\
& \leq c_{2}^{-1 / 2}\|\mathfrak{E} f\|_{\mathcal{N}_{\Phi}} \\
& \leq\left(c_{1} c_{2}\right)^{-1 / 2}\|\mathfrak{E} f\|_{W_{2}^{\tau}\left(\mathbb{R}^{n}\right)} \\
& \leq\left(c_{1} c_{2}\right)^{-1 / 2}\|\mathfrak{E}\|\|f\|_{W_{2}^{\tau}(\Omega)} .
\end{aligned}
$$

Setting $C_{\Omega, \Phi}:=\left(c_{1} c_{2}\right)^{-1 / 2}\|\mathfrak{E}\|$ completes the proof.

We now employ this lemma and the results obtained in the previous section to derive bounds on $f-I_{X} f$, in both continuous and discrete norms, for the case $p=2$.

Proposition 3.2. Suppose $\tau=k+s$, where $k$ is a positive integer and $0<s \leq 1$. Let $\alpha$ be a multi-index satisfying $k>|\alpha|+n / 2$, and let $X \subset \Omega$ be a discrete set with mesh norm $h$ satisfying (91). If $f \in W_{2}^{\tau}(\Omega)$ and if $1 \leq q \leq \infty$, then

$$
\left|f-I_{X} f\right|_{W_{q}^{|\alpha|}(\Omega)} \leq C_{k, n, q,|\alpha|, \Omega, \Phi} h^{\tau-|\alpha|-n(1 / 2-1 / q)_{+}}\|f\|_{W_{2}^{\tau}(\Omega)} .
$$

In addition, the continuous least squares error $(q=2)$ satisfies the bound,

$$
\min _{v \in V_{X, \Phi}}\|f-v\|_{L_{2}(\Omega)} \leq C_{k, n, \Omega, \Phi} h^{\tau}\|f\|_{W_{2}^{\tau}(\Omega)} .
$$

Proof. Apply Theorem 2.12 to $u=f-I_{X} f$, with $p=2$. Using Lemma 3.1 then gives us (18). Since $I_{X} f \in V_{X, \Phi}$, we also have that

$$
\min _{v \in V_{X, \Phi}}\|f-v\|_{L_{2}(\Omega)} \leq\left\|f-I_{X} f\right\|_{L_{2}(\Omega)} .
$$

The estimate (19) then follows from (18) with $q=2$ and $|\alpha|=0$.

The case where the discrete norm is to be bounded, rather than the continuous one, can be dealt with in a similar way. 
Proposition 3.3. Suppose $\tau=k+s$, where $k$ is a positive integer and $0<s \leq 1$. Let $\alpha$ be a multi-index satisfying $k>|\alpha|+n / 2$. Also, let $X \subset \Omega$ be a discrete set with mesh norm $h=h_{X}$ satisfying (9). Let $Y=\left\{y_{1}, \ldots, y_{M}\right\} \subset \Omega$ be a second discrete set, with $h_{Y} \leq h$. If $f \in W_{2}^{\tau}(\Omega)$, and if $1 \leq q \leq \infty$, then

$$
\left|f-I_{X} f\right|_{w_{q}^{|\alpha|}(Y)} \leq C_{k, n, q,|\alpha|, \Omega, \Phi} \rho_{Y}^{n / q} h^{\tau-|\alpha|-n(1 / 2-1 / q)_{+}}\|f\|_{W_{2}^{\tau}(\Omega)},
$$

where $|\cdot|_{w_{q}^{|\alpha|}(Y)}$ is defined in (12). Also, the discrete least squares error satisfies the bound,

$$
\min _{v \in V_{X, \Phi}}\|f-v\|_{\ell_{2}(Y)} \leq C_{k, n, \Omega, \Phi} \rho_{Y}^{n / 2} h^{\tau}\|f\|_{W_{2}^{\tau}(\Omega)}
$$

Proof. Apply Theorem [2.13 to $u=f-I_{X} f$. Using Lemma 3.1 with $p=2$, then completes the proof. Again, because $I_{X} f \in V_{X, \Phi}$, we have that

$$
\min _{v \in V_{X, \Phi}}\|f-v\|_{\ell_{2}(Y)} \leq\left\|f-I_{X} f\right\|_{\ell_{2}(Y)} .
$$

The estimate (22) then follows from the interpolation estimate (21) with $|\alpha|=0$ and $q=2$.

We remark that in both cases the interpolant is a good approximation to the least squares fit.

3.1.2. Thin-plate splines. The RBFs we just discussed are all positive definite functions. The thin-plate splines, however, are RBFs that are conditionally positive definite functions. If $k>n / 2$ is an integer, then we define the thin-plate spline corresponding to $n$ and $k$ as follows. For $\|x\|_{2} \neq 0$, we let

$$
\Phi_{n, k}(x):=c_{n, k} \begin{cases}\|x\|_{2}^{2 k-n} & \text { for } n \text { odd, } \\ \|x\|_{2}^{2 k-n} \log \|x\|_{2} & \text { for } n \text { even, }\end{cases}
$$

where $c_{n, k}$ is a constant chosen so that $\Phi_{n, k}$ is a fundamental solution of the iterated Laplacian. In terms of the distributional Fourier transform, this is equivalent to requiring that $\widehat{\Phi}_{n, k}(\omega)=\|\omega\|_{2}^{-2 k}$, if $\omega \neq 0$.

The native space associated with $\Phi_{n, k}$ is the Beppo-Levi space,

$$
\mathrm{BL}_{k}\left(\mathbb{R}^{n}\right):=\left\{f \in C\left(\mathbb{R}^{n}\right): D^{\alpha} f \in L_{2}\left(\mathbb{R}^{n}\right) \text { for all }|\alpha|=k\right\},
$$

which is equipped with the semi-inner product

$$
(f, g)_{\mathrm{BL}_{k}\left(\mathbb{R}^{n}\right)}=\sum_{|\alpha|=k} \frac{k !}{\alpha !}\left(D^{\alpha} f, D^{\alpha} g\right)_{L_{2}\left(\mathbb{R}^{n}\right)}
$$

and induced semi-norm $|\cdot|_{\mathrm{BL}_{k}\left(\mathbb{R}^{n}\right)}$. For Beppo-Levi spaces on $\Omega$, similar definitions apply. Both the semi-norm $|\cdot|_{\mathrm{BL}_{k}\left(\mathbb{R}^{n}\right)}$ and $|\cdot|_{\mathrm{BL}_{k}(\Omega)}$ are equivalent to the corresponding Sobolev semi-norms of order $k$.

An interpolant $I_{X} f$, which is associated with $\Phi_{n, k}$ and $\left.f\right|_{X}$ from a continuous function $f$, includes a polynomial piece $p \in \pi_{k-1}\left(\mathbb{R}^{n}\right)$ as well as a linear combination of $\operatorname{span}\left\{\Phi_{n, k}\left(x-x_{j}\right)\right\}_{j=1}^{N}$. That is, $I_{X} f$ is in

$$
V_{X, n, k}=\pi_{k-1}\left(\mathbb{R}^{n}\right) \oplus \operatorname{span}\left\{\Phi_{n, k}\left(x-x_{j}\right)\right\}_{j=1}^{N} .
$$

To insure that the interpolant exists, one must make the additional assumption that the finite set $X \subset \Omega$ is unisolvent for $\pi_{k-1}\left(\mathbb{R}^{n}\right)$. Under this assumption, the method reproduces polynomials in $\pi_{k-1}\left(\mathbb{R}^{n}\right)$. In addition, if $f$ is in the native space 
$\mathrm{BL}_{k}\left(\mathbb{R}^{n}\right)$, then $I_{X} f$ minimizes the semi-norm $|f-v|_{\mathrm{BL}_{k}\left(\mathbb{R}^{n}\right)}$ among all $v \in V_{X, n, k}$. As in the previous section, this implies that

$$
\left|f-I_{X} f\right|_{\mathrm{BL}_{k}\left(\mathbb{R}^{n}\right)} \leq|f|_{\mathrm{BL}_{k}\left(\mathbb{R}^{n}\right)}, \quad f \in \mathrm{BL}_{k}\left(\mathbb{R}^{n}\right) .
$$

Now, an extension theorem of Duchon [5 Lemma 3.1] shows the existence of a linear map $\mathfrak{E}_{k}: W_{2}^{k}(\Omega) \rightarrow B L_{k}\left(\mathbb{R}^{n}\right)$ such that for all $f \in W_{2}^{k}(\Omega)$ we have $\left.\mathfrak{E}_{k} f\right|_{\Omega}=f$ and $\left|\mathfrak{E}_{k} f\right|_{B L_{k}\left(\mathbb{R}^{n}\right)} \leq \| \mathfrak{E}_{k}|||f|_{\mathrm{BL}_{k}(\Omega)}$. Essentially repeating the proof of our own Lemma 3.1 then yields the following:

Lemma 3.4. Let $k>n / 2$ be an integer, $X=\left\{x_{1}, x_{2}, \ldots, x_{N}\right\} \subset \Omega$ be unisolvent for $\pi_{k-1}\left(\mathbb{R}^{n}\right)$, and let $f \in W_{2}^{k}(\Omega)$. If $I_{X} f \in V_{X, n, k}$ is the unique function that interpolates $f$ on $X$, then there exists a constant $C_{\Omega, n, k}$ such that

$$
\left|f-I_{X} f\right|_{W_{2}^{k}(\Omega)} \leq C_{\Omega, n, k}|f|_{W_{2}^{k}(\Omega)} \leq C_{\Omega, n, k}\|f\|_{W_{2}^{k}(\Omega)} .
$$

Recall that a finite, discrete set $X \subset \mathbb{R}^{n}$ is unisolvent for $\pi_{k-1}\left(\mathbb{R}^{n}\right)$ if for $p \in$ $\pi_{k-1}\left(\mathbb{R}^{n}\right)$ the vanishing of $p$ on $X$-i.e., $\left.p\right|_{X}=0$-implies that $p \equiv 0$. Suppose that we again have $X \subset \Omega$, with mesh norm $h$ satisfying (9). We want to show that under these conditions we have the slightly stronger result that $X$ is unisolvent with respect to $\pi_{k}\left(\mathbb{R}^{n}\right)$.

Proposition 3.5. Let $k \geq 1$ be an integer. If $X$ a finite, discrete subset of $X$, with mesh norm $h$ satisfying (9), then $X$ is unisolvent for $\pi_{k}\left(\mathbb{R}^{n}\right)$.

Proof. In Theorem 2.12 take $s=1,|\alpha|=0, q=\infty, p=2 n$. If $u$ is a polynomial in $\pi_{k}\left(\mathbb{R}^{n}\right)$, with $\left.u\right|_{X}=0$, then, we have that $\|u\|_{L_{\infty}(\Omega)} \leq C h^{k+1 / 2}|u|_{W_{2 n}^{k+1}(\Omega)}$. Since $D^{\alpha} u \equiv 0$ for $|\alpha| \geq k+1$, the norm $|u|_{W_{2 n}^{k+1}(\Omega)}=0$. It then follows from Theorem 2.12 that $\left.u\right|_{\Omega}=0$ and, since $\Omega$ contains open sets, that $u \equiv 0$. Thus, $X$ is unisolvent for $\pi_{k}\left(\mathbb{R}^{n}\right)$.

By our remarks above, the set $X$ being unisolvent implies that for any $f \in C(\Omega)$, there is a unique interpolant $I_{X} f \in V_{X, n, k}$ for $f$. This plus the lemma above is precisely what we require to get the same type of estimates that we obtained in the last section. In fact, repeating the proofs of Propositions 3.2 and 3.3 yields the same estimates. We formally state these observations below.

Corollary 3.6. Under the assumptions on $X, \Omega$, and $h$ made in Propositions 3.2 and [3.3, the interpolant $I_{X} f \in V_{X, n, k}$ exists and is unique. Moreover, the estimates in both propositions also hold for $I_{X} f \in V_{X, n, k}$.

3.2. Error estimates for functions outside of $\mathcal{N}_{\Phi}$. It is now our goal to establish discrete and continuous Sobolev-type error estimates for functions that are outside the native space, but still in a Sobolev space or a $C^{k}$-space. More precisely, if we let $\tau$ determine the decay of $\widehat{\Phi}$, we will assume either that $f \in W_{2}^{t}(\Omega)$, where $\tau \geq t>n / 2$, or that $f \in C^{k}(\bar{\Omega}), \tau \geq k>n / 2$.

3.2.1. Approximation error estimates. For approximation rather than interpolation, such error estimates have been derived for integer $\tau$ in [16], using a technique introduced in [13]. We will extend this result to positive, real $\tau$. The proof we give here is simpler than that given in [16]; it is based upon recent results from [10]. 
Lemma 3.7 ([10, Proposition 3.7]). Let $t \geq r \geq 0$. If $f \in W_{2}^{t}\left(\mathbb{R}^{n}\right)$, then there exists a constant $c_{t, r}$ such that for every $\sigma>0$ we can choose a band limited function $g_{\sigma} \in \mathcal{B}_{\sigma}=\left\{f \in L_{2}\left(\mathbb{R}^{n}\right): \operatorname{supp}(\widehat{f}) \subseteq B(0, \sigma)\right\}$ with

$$
\left\|f-g_{\sigma}\right\|_{W_{2}^{r}\left(\mathbb{R}^{n}\right)} \leq c_{t, r} \sigma^{r-t}\|f\|_{W_{2}^{t}\left(\mathbb{R}^{n}\right)} .
$$

Obviously, this result is important mainly in the case of $\sigma>1$, and in such a situation we will use it now.

Theorem 3.8. Suppose $\Phi$ is a positive definite function satisfying (15), with $\tau \geq$ $t>n / 2$, and that $X=\left\{x_{1}, \ldots, x_{N}\right\} \subset \Omega$ has mesh norm $h$ satisfying (9). If $f \in W_{2}^{t}(\Omega)$, then there exists a function $v \in V_{X, \Phi}=\operatorname{span}\left\{\Phi\left(\cdot-x_{j}\right): x_{j} \in X\right\}$ such that for every real $0 \leq r \leq t$,

$$
\|f-v\|_{W_{2}^{r}(\Omega)} \leq C h^{t-r}\|f\|_{W_{2}^{t}(\Omega)} .
$$

Here, $C$ is a constant independent of $f$ and $h$.

Proof. Let $\mathfrak{E}$ be the extension operator discussed in the proof of Lemma 3.1. We first extend the function $f \in W_{2}^{t}(\Omega)$ to a function $\mathfrak{E} f \in W_{2}^{t}\left(\mathbb{R}^{n}\right)$. Next we pick a band limited function $g_{\sigma}$ that approximates $\mathfrak{E} f$ according to (24), with $\sigma=1 / h$. Finally, we let $v=I_{X} g_{\sigma}$. Then, we have

$$
\begin{aligned}
\|f-v\|_{W_{2}^{r}(\Omega)} & \leq\left\|\mathfrak{E} f-g_{\sigma}\right\|_{W_{2}^{r}\left(\mathbb{R}^{n}\right)}+\left\|g_{\sigma}-I_{X} g_{\sigma}\right\|_{W_{2}^{r}(\Omega)} \\
& \leq c_{1} h^{t-r}\|\mathfrak{E} f\|_{W_{2}^{t}\left(\mathbb{R}^{n}\right)}+c_{2} h^{\tau-r}\left\|g_{\sigma}\right\|_{W_{2}^{\tau}(\Omega)} \\
& \leq c_{3} h^{t-r}\|f\|_{W_{2}^{t}(\Omega)}+c_{2} h^{\tau-r}\left\|g_{\sigma}\right\|_{W_{2}^{\tau}(\Omega)},
\end{aligned}
$$

where we have used (24), Proposition 3.2, and the continuity of the extension operator $\mathfrak{E}$. To estimate the second term on the right, we observe that $\left\|g_{\sigma}\right\|_{W_{2}^{\tau}(\Omega)} \leq$ $\left\|g_{\sigma}\right\|_{W_{2}^{\tau}\left(\mathbb{R}^{n}\right)}$. Now, $g_{\sigma}$ is band-limited, and so $\left\|g_{\sigma}\right\|_{W_{2}^{\tau}\left(\mathbb{R}^{n}\right)} \leq c \sigma^{\tau-t}\left\|g_{\sigma}\right\|_{W_{2}^{t}\left(\mathbb{R}^{n}\right)}=$ $c h^{t-\tau}\left\|g_{\sigma}\right\|_{W_{2}^{t}\left(\mathbb{R}^{n}\right)}$. (This is trivial to show for $p=2$. It is, of course, a special case of Bernstein's Theorem for functions of exponential type.) Another application of (24) and the continuity of $\mathfrak{E}$ establishes

$$
\left\|g_{\sigma}\right\|_{W_{2}^{t}\left(\mathbb{R}^{n}\right)} \leq\|\mathfrak{E} f\|_{W_{2}^{t}\left(\mathbb{R}^{n}\right)}+\left\|\mathfrak{E} f-g_{\sigma}\right\|_{W_{2}^{t}\left(\mathbb{R}^{n}\right)} \leq c_{4}\|\mathfrak{E} f\|_{W_{2}^{t}\left(\mathbb{R}^{n}\right)} \leq c_{5}\|f\|_{W_{2}^{t}(\Omega)} .
$$

Combining these bounds results in $\left\|g_{\sigma}\right\|_{W_{2}^{\tau}(\Omega)} \leq c_{5} h^{t-\tau}\|f\|_{W_{2}^{t}(\Omega)}$. Overall, this gives us the estimate,

$$
\|f-v\|_{W_{2}^{r}(\Omega)} \leq\left(c_{3} h^{t-r}+c_{2} c_{5} h^{\tau-r} h^{t-\tau}\right)\|f\|_{W_{2}^{t}(\Omega)} \leq C h^{t-r}\|f\|_{W_{2}^{t}(\Omega)},
$$

which is what we wished to show.

3.2.2. Interpolation error estimates. We now turn to error estimates for interpolation of a function $f$ in $W_{2}^{k}(\Omega)$ by the smoother functions in $V_{X, \Phi} \subset \mathcal{N}_{\Phi}$. In the special case of interpolation by means of an integer order thin-plate spline, Brownlee and Light 2] have obtained $L_{p}$ error estimates in terms of $|f|_{W_{2}^{k}(\Omega)}$. We will treat the general RBF case here, but we will need to work in the space $C^{k}(\bar{\Omega})$, rather than $W_{2}^{k}(\Omega)$.

We begin with a few remarks about the extension operator $\mathfrak{E}$ constructed by Stein [14 §VI.3]. Stein explicitly states that this operator maps $W_{p}^{k}(\Omega)$ boundedly to $W_{p}^{k}\left(\mathbb{R}^{n}\right)$, for $1 \leq p \leq \infty$ and for any integer $k \geq 0$. In fact, it does a little more 
than that. If $f \in C^{k}(\bar{\Omega})$, then Stein's construction yields $\mathfrak{E} f \in C^{k}\left(\mathbb{R}^{n}\right) \cap W_{\infty}^{k}\left(\mathbb{R}^{n}\right)$. Moreover, if $k>n / 2$, then the fact that $\mathfrak{E} f \in W_{2}^{k}\left(\mathbb{R}^{n}\right)$ also implies that $\widehat{\mathfrak{E} f} \in$ $L^{1}\left(\mathbb{R}^{n}\right)$, which in turn yields $\lim _{|x| \rightarrow \infty} \mathfrak{E} f(x)=0$. Of course, we also have the norm bounds

$$
\|\mathfrak{E} f\|_{W_{2}^{k}\left(\mathbb{R}^{n}\right)} \leq C_{1}\|f\|_{W_{2}^{k}(\Omega)} \text { and }\|\mathfrak{E} f\|_{W_{\infty}^{k}\left(\mathbb{R}^{n}\right)} \leq C_{2}\|f\|_{C^{k}(\bar{\Omega})} .
$$

Since $\|f\|_{W_{2}^{k}(\Omega)} \leq C_{3}\|f\|_{C^{k}(\bar{\Omega})}$, we have that

$$
\max \left\{\|\mathfrak{E} f\|_{W_{2}^{k}\left(\mathbb{R}^{n}\right)},\|\mathfrak{E} f\|_{W_{\infty}^{k}\left(\mathbb{R}^{n}\right)}\right\} \leq C\|f\|_{C^{k}(\bar{\Omega})} .
$$

We will now make use of the extension $\mathfrak{E} f$ to obtain a band-limited interpolant to $f$ on $X \subset \Omega$. For normalization purposes we will require $\operatorname{diam}(X) \leq 1$.

Lemma 3.9. Let $f \in C^{k}(\bar{\Omega})$ and suppose that $X=\left\{x_{1}, x_{2}, \ldots, x_{N}\right\} \subset \Omega$ satisfies $\operatorname{diam}(X) \leq 1$. Let $q_{X}$ be the separation radius of $X$. Then, there is a constant $c_{n}$, depending only on the dimension $n$, such that, for any $\sigma \geq \frac{c_{n}}{q_{X}}$, there exists a band-limited function $f_{\sigma} \in \mathcal{B}_{\sigma}$ for which

$$
\left.f\right|_{X}=\left.f_{\sigma}\right|_{X} \text { and }\left\|f_{\sigma}\right\|_{W_{2}^{k}\left(\mathbb{R}^{n}\right)} \leq C\|f\|_{C^{k}(\bar{\Omega})} .
$$

Proof. The extension $\mathfrak{E} f$ is in $C_{0}\left(\mathbb{R}^{n}\right) \cap L_{2}\left(\mathbb{R}^{n}\right)$, so [10] Theorem 3.5] gives us the existence of $f_{\sigma}$ for which $\left.\mathfrak{E} f\right|_{X}=\left.f_{\sigma}\right|_{X}$. Since $\left.\mathfrak{E} f\right|_{\Omega}=f$, we see that $\left.f\right|_{X}=\left.f_{\sigma}\right|_{X}$. In addition, since $\mathfrak{E} f \in W_{2}^{k}\left(\mathbb{R}^{n}\right) \cap W_{\infty}^{k} \cap C^{k}\left(\mathbb{R}^{n}\right)$, [10, Proposition 3.12] provides the estimate

$$
\left\|f_{\sigma}\right\|_{W_{2}^{k}\left(\mathbb{R}^{n}\right)} \leq \max \left\{\|\mathfrak{E} f\|_{W_{2}^{k}\left(\mathbb{R}^{n}\right)},\|\mathfrak{E} f\|_{W_{\infty}^{k}\left(\mathbb{R}^{n}\right)}\right\} .
$$

Applying (25) to bound the right side above then yields (26), which completes the proof.

Theorem 3.10. Let $k$ and $j$ be integers, with $0 \leq j<k \leq \tau$ and $k>n / 2$, and let $f \in C^{k}(\bar{\Omega})$. Also suppose that $X=\left\{x_{1}, x_{2}, \ldots, x_{N}\right\} \subset \Omega$ satisfies $\operatorname{diam}(X) \leq 1$, with mesh norm $h$ satisfying (9). Then,

$$
\left|f-I_{X} f\right|_{W_{q}^{j}(\Omega)} \leq C \rho_{X}^{\tau-k} h^{k-j-n(1 / 2-1 / q)_{+}}\|f\|_{C^{k}(\bar{\Omega})},
$$

where $\rho_{X}=\frac{h}{q_{X}}$ is the mesh ratio for $X$ in $\Omega$.

Proof. By Theorem 2.12, we have

$$
\left|f-I_{X} f\right|_{W_{q}^{j}(\Omega)} \leq C h^{k-j-n(1 / 2-1 / q)+}\left|f-I_{X} f\right|_{W_{2}^{k}(\Omega)} .
$$

Choosing $\sigma=\frac{c_{n}}{q_{X}}$ in Lemma 3.9 we have the existence of $f_{\sigma} \in \mathcal{B}_{\sigma}$ that interpolates $f$ on $X$. Recall that the interpolation operator $I_{X}$ depends only on $\left.f\right|_{X}=\left.f_{\sigma}\right|_{X}$, so $I_{X} f=I_{X} f_{\sigma}$. Consequently, we have this chain of inequalities:

$$
\begin{aligned}
\left|f-I_{X} f\right|_{W_{2}^{k}(\Omega)} & =\left|f-I_{X} f_{\sigma}\right|_{W_{2}^{k}(\Omega)} \\
& \leq\left|f-f_{\sigma}\right|_{W_{2}^{k}(\Omega)}+\left|f_{\sigma}-I_{X} f_{\sigma}\right|_{W_{2}^{k}(\Omega)} \\
& \leq|f|_{W_{2}^{k}(\Omega)}+\left|f_{\sigma}\right|_{W_{2}^{k}(\Omega)}+\left|f_{\sigma}-I_{X} f_{\sigma}\right|_{W_{2}^{k}(\Omega)} .
\end{aligned}
$$

By Proposition 3.2 (or, for the thin-plate splines, Corollary [3.6), with $f$ replaced by $f_{\sigma}, q$ by 2 , and so on, we have

$$
\left|f_{\sigma}-I_{X} f_{\sigma}\right|_{W_{2}^{k}(\Omega)} \leq C h^{\tau-k}\left\|f_{\sigma}\right\|_{W_{2}^{\tau}(\Omega)} .
$$


Obviously, $\left|f_{\sigma}\right|_{W_{2}^{\tau}(\Omega)} \leq\left\|f_{\sigma}\right\|_{W_{2}^{\tau}\left(\mathbb{R}^{n}\right)}$. By Bernstein's inequality for functions of exponential type, $\left\|f_{\sigma}\right\|_{W_{2}^{\tau}\left(\mathbb{R}^{n}\right)} \leq c \sigma^{\tau-k}\left\|f_{\sigma}\right\|_{W_{2}^{k}\left(\mathbb{R}^{n}\right)}$. Hence, we have

$$
\left|f-I_{X} f\right|_{W_{2}^{k}(\Omega)} \leq|f|_{W_{2}^{k}(\Omega)}+\left(1+C h^{\tau-k} \sigma^{\tau-k}\right)\left\|f_{\sigma}\right\|_{W_{2}^{k}\left(\mathbb{R}^{n}\right)} .
$$

However, $\sigma=\frac{c_{n}}{q_{X}}$, so

$$
\left|f-I_{X} f\right|_{W_{2}^{k}(\Omega)} \leq|f|_{W_{2}^{k}(\Omega)}+\left(1+C c_{n}^{\tau-k} \rho_{X}^{\tau-k}\right)\left\|f_{\sigma}\right\|_{W_{2}^{k}\left(\mathbb{R}^{n}\right)},
$$

where we recall that $\rho_{X}=\frac{h}{q_{X}} \geq 1$ is the mesh ratio for $X$ in $\Omega$. By (26), $\left\|f_{\sigma}\right\|_{W_{2}^{k}(\Omega)} \leq C\|f\|_{C^{k}(\bar{\Omega})}$. In addition, we have the standard estimate $|f|_{W_{2}^{k}(\Omega)} \leq$ $C^{\prime}\|f\|_{C^{k}(\bar{\Omega})}$. Combining all of these and simplifying, we obtain

$$
\begin{aligned}
\left|f-I_{X} f\right|_{W_{2}^{k}(\Omega)} & \leq\left(1+C^{\prime}+C c_{n}^{\tau-k} \rho_{X}^{\tau-k}\right)\|f\|_{C^{k}(\bar{\Omega})} \\
& \leq C^{\prime \prime} \rho_{X}^{\tau-k}\|f\|_{C^{k}(\bar{\Omega})} .
\end{aligned}
$$

Using this bound in (28) then gives us (27), which completes the proof.

Our final result is a corollary that deals with the discrete case, rather than the continuous one.

Corollary 3.11. Let $k$ and $j$ be integers, with $0 \leq j<k \leq \tau$ and $k>n / 2$, and let $f \in C^{k}(\bar{\Omega})$. Also suppose that $X=\left\{x_{1}, x_{2}, \ldots, x_{N}\right\} \subset \Omega$ satisfies $\operatorname{diam}(X) \leq 1$, with mesh norm $h$ satisfying (91). In addition, let $Y$ be a second discrete set, with $h_{Y} \leq h$. Then,

$$
\left|f-I_{X} f\right|_{w_{q}^{j}(Y)} \leq C \rho_{Y}^{n / q} \rho_{X}^{\tau-k} h^{k-j-n(1 / 2-1 / q)+}\|f\|_{C^{k}(\bar{\Omega})},
$$

where the discrete norm on the left above is defined in (11).

Proof. The proof is nearly identical to the theorem above. The difference is that at the start one needs to use Theorem 2.13, which is the discrete version of Theorem 2.12,

\section{REFERENCES}

[1] S. C. Brenner and L. R. Scott, The mathematical theory of finite element methods, Springer, New York, 1994. MR 95f:65001

[2] R. Brownlee and W. Light, Approximation orders for interpolation by surface splines to rough functions, IMA J. Numer. Anal., 24 (2004), 179-192.

[3] C. de Boor, R. A. DeVore, and A. Ron, Approximation from shift-invariant subspaces of $L_{2}\left(\mathbb{R}^{d}\right)$, Trans. Amer. Math. Soc., 341 (1994), 787-806. MR 94d:41028

[4] R. A. DeVore and R. C. Sharpley, Besov spaces on domains in $\mathbb{R}^{d}$, Trans. Amer. Math. Soc., 335 (1993), 843-864. MR 93d:46051

[5] J. Duchon, Sur l'erreur d'interpolation des fonctions de plusieurs variables par les $D^{m}$. splines, Rev. Française Automat. Informat. Rech. Opér. Anal. Numer., 12 (1978), 325-334. MR 80j:41052

[6] W. R. Madych and S. A. Nelson, Multivariate interpolation and conditionally positive definite functions, Approximation Theory Appl., 4 (1988), 77-89. MR 90e:41006

[7] _ Multivariate interpolation and conditionally positive definite functions II, Math. Comp., 54 (1990), 211-230. MR 90e:41007

[8] W. R. Madych and E. H. Potter, An estimate for multivariate interpolation, J. Approx. Theory, 43 (1985), 132-139. MR 86g:65022

[9] C. A. Micchelli, Interpolation of scattered data: Distance matrices and conditionally positive definite functions, Constr. Approx., 2 (1986), 11-22. MR 88d:65016

[10] F. J. Narcowich and J. D. Ward, Scattered-data interpolation on $\mathbb{R}^{n}$ : Error estimates for radial basis and band-limited functions, SIAM J. Math. Anal., to appear. 
[11] F. J. Narcowich, J. D. Ward, and H. Wendland, Refined error estimates for radial basis function interpolation, Constr. Approx., 19 (2003), 541-564.

[12] A. Ron, The $L_{2}$-approximation orders of principal shift-invariant spaces generated by a radial basis function, Numerical Methods in Approximation Theory. Vol. 9: Proceedings of the conference held in Oberwolfach, Germany, November 24-30, 1991 (Basel) (D. Braess et al., eds.), Int. Ser. Numer. Math., vol. 105, Birkhäuser, 1992, pp. 245-268. MR 95d:41035

[13] R. Schaback, Approximation by radial basis functions with finitely many centers, Constr. Approx., 12 (1996), 331-340. MR 97d:41013

[14] E. M. Stein, Singular Integrals and Differentiability Properties of Functions, Princeton University Press, Princeton, New Jersey, 1971. MR 44:7280

[15] H. Wendland, Piecewise polynomial, positive definite and compactly supported radial functions of minimal degree, Adv. Comp. Math., 4 (1995), 389-396. MR 96h:41025

[16] _ Meshless Galerkin methods using radial basis functions, Math. Comp., 68 (1999), 1521-1531. MR 99m:65221

[17] _ Local polynomial reproduction and moving least squares approximation, IMA J. Numer. Anal., 21 (2001), 285-300. MR 2002a:65025

Department of Mathematics, Texas A\&M University, College Station, Texas 778433368

E-mail address: fnarc@math.tamu.edu

Department of Mathematics, Texas A\&M University, College Station, Texas 778433368

E-mail address: jward@math.tamu.edu

Universität Göttingen, Lotzestrasse 16-18, D-37083, Göttingen, Germany

E-mail address: wendland@math.uni-goettingen.de 